\title{
A split-face study evaluating the efficacy of a topical antioxidant cream containing tocotrienol after 1064-nm picosecond Nd:YAG laser treatment for environment-induced skin pigmentation
}

Moon Seok Kang ${ }^{1}$, Jun Hyun Kim ${ }^{2}$, Seung Min Nam², Eun Soo Park ${ }^{2}$

${ }^{1}$ WONJIN Plastic Surgery Clinic, Seoul; ${ }^{2}$ Department of Plastic and Reconstructive Surgery, Soonchunhyang University College of Medicine, Bucheon, Korea

This work was supported by the Soonchunhyang University Research Fund.
Background This study aimed to evaluate the efficacy and safety of a topical antioxidant cream containing tocotrienol after 1064-nm picosecond neodymium-doped yttrium aluminum garnet (Nd:YAG) laser treatment for environment-induced skin pigmentation.

Methods A split-face study was conducted between December 2020 and March 2021 in 26 patients ( 25 women and one man) who were treated with a 1064-nm picosecond $\mathrm{Nd}$ :YAG laser. On the right side of the face, an antioxidant cream containing tocotrienol was applied after picosecond laser treatment, and on the left side, only picosecond laser treatment was performed. We compared the right and left sides of the face at 4 weeks after laser treatment using an automatic skin analysis device to investigate skin pigmentation. The melasma severity scores of the two sides were evaluated before and 4 weeks after picosecond laser treatment.

Results The skin pigmentation score showed a significant difference between pretreatment and 4 weeks after picosecond laser treatment on both sides of the face $(\mathrm{P}<0.001)$, and there was a significant difference in skin pigmentation score improvement between the right and left sides $(P<0.001)$. The melasma severity score showed a significant difference on the right side between pretreatment and 4 weeks after laser treatment $(P<0.001)$, but there was no significant change on the left side $(P>0.05)$. Conclusions Our results show that, for the treatment of environment-induced skin pigmentation, the application of a tocotrienol-containing antioxidant cream after 1064-nm picosecond laser treatment can provide good results without any complications.

Keywords Laser / Pigmentation / Antioxidants
Received: Jun 25, 2021 Revised: Jul 6, 2021 Accepted: Jul 8, 2021 Correspondence: Seung Min Nam Department of Plastic and Reconstructive, Soonchunhyang University Bucheon Hospital, Soonchunhyang University College of Medicine, 170 Jomaru-ro, Wonmi-gu, Bucheon 14584, Korea Tel: +82-32-621-5311, Fax: +82-32-621-5316

E-mail: zodiac1003@schmc.ac.kr

Copyright @ 2021 The Korean Society for Aesthetic Plastic Surgery.

This is an Open Access article distributed under the terms of the Creative Commons Attribution Non-Commercial License (https://creativecommons.org/licenses/by-nc/4.0/) which permits unrestricted non-commercial use, distribution, and reproduction in any medium, provided the original work is properly cited. $\quad$ www.e-aaps.org

\section{INTRODUCTION}

Several environmental factors, including ultraviolet (UV) radiation, can induce skin pigmentation and an inflammatory response, which may then provoke degradation of the extracellular matrix [1]. Facial skin pigmentation is often associated with depressive emotional changes and psychological stress in patients [2-4]. Solar lentigo is the most common skin pigmentation lesion resulting from environment-induced skin pigmentation, and presents as a well-demarcated, pale-to-dark brown macule characterized by local melanocyte proliferation and melanin accumulation in the skin $[4,5]$. 
The 1064-nm Q-switched neodymium-doped yttrium aluminum garnet (Nd:YAG) laser has been used to treat skin pigmentation lesions, and satisfactory results have been reported in several studies [6-9]. Despite these satisfactory results, the outcomes of 1064-nm Nd:YAG laser treatment can be limited and unpredictable, and adverse effects include pain and skin hypopigmentation and hyperpigmentation [10]. Recently, a picosecond laser system was developed to minimize adverse effects and maximize the beneficial effects. The picosecond laser has a short pulse duration, from a billionth to a trillionth of a second [11]. Because of the short pulse duration, the picosecond laser can induce a largely photomechanical effect on the target tissue to enable satisfactory results while minimizing damage to the surrounding tissues from the photothermal effect [12-14].

Vitamin E can scavenge free radicals and has been reported to have anti-melanogenic effects [15]. Tocopherol and tocotrienol, which are vitamin $\mathrm{E}$ analogues, can be introduced as effective ingredients in whitening cosmetics to improve skin pigmentation [16]. In particular, tocotrienol are believed to be effective in skin regeneration and renewal due to their antioxidant properties [17].

The primary objective of this study was to evaluate the efficacy and safety of a topical antioxidant cream containing tocotrienol after 1064-nm picosecond Nd:YAG laser application for the treatment of environment-induced skin pigmentation.

\section{METHODS}

We conducted a split-face study to evaluate the efficacy of an antioxidant cream containing tocotrienol after picosecond 1064-nm Nd:YAG laser treatment. Of the patients who underwent picosecond 1064-nm Nd:YAG laser treatment for environment-induced

Table 1. Demographic characteristics of patients

\begin{tabular}{lc}
\hline Variable & Value $(\mathrm{n}=26)$ \\
\hline Sex, No. (\%) & \\
Male & $1(3.8)$ \\
Female & $25(96.2)$ \\
Age lyr), mean (range) & $43.1(37-55)$ \\
Fitzpatrick skin type, No. (\%) & \\
III & $4(15.4)$ \\
IV & $22(84.6)$ \\
\hline
\end{tabular}
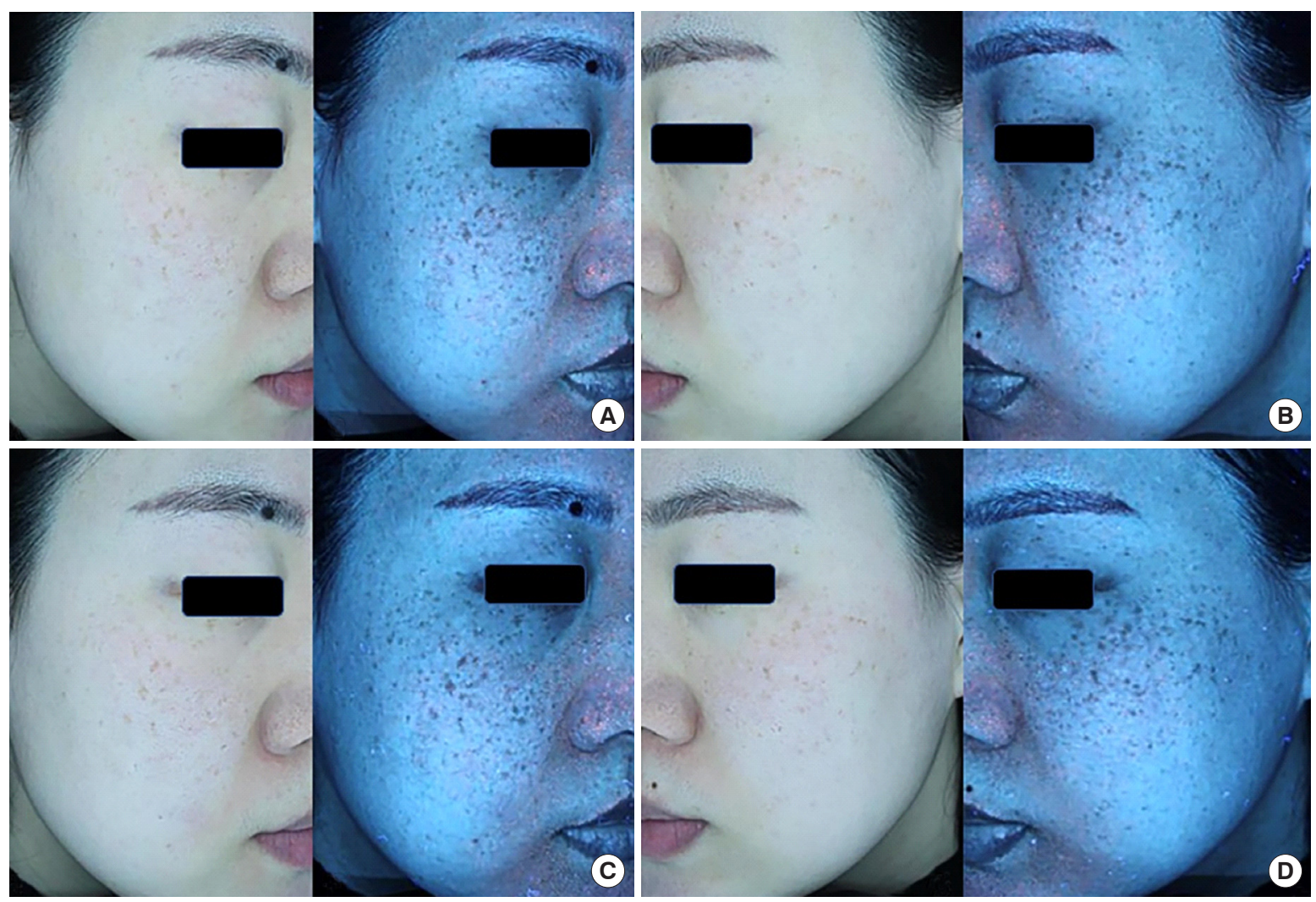

Fig. 1. Clinical and ultraviolet photographs of a 43-year-old female patient with environment-induced skin pigmentation. (A) The right side of the face, to which a combination of picosecond laser and antioxidant cream containing tocotrienol was applied. (B) The left side of the face, to which only picosecond laser treatment was applied. (C) The right side of the face at 4 weeks after laser treatment. (D) The left side of the face at 4 weeks after laser treatment. 
skin pigmentation between December 2020 and March 2021, 26 patients (Fitzpatrick skin type III-IV), who understood and agreed with the rationale and methodology of this study were enrolled. We excluded patients who had a history of keloid scar, recent use of oral retinoids, pregnancy, immunosuppressive drug use, active systemic or local infections, or psychiatric illness.

The study conformed to the principles of the Declaration of Helsinki, and written consent was obtained from each patient both for surgery and the publication of photographs of the results. The study was approved by the Institutional Review Board of Soonchunhyang University Bucheon Hospital (IRB No. 2021-06-015).

\section{Laser treatment}

A topical 5\% lidocaine anesthetic ointment (Emla; AstraZeneca AB, Karlskoga, Sweden) was administered to the target area for laser treatment. After 30 minutes to 1 hour of application, the patients washed off the ointment with mild soap and water immediately before the laser treatment. All patients were treated using a 1064$\mathrm{nm}$ picosecond Nd:YAG laser (Picocare; Wontech, Daejeon, Korea), with the settings of a $7-\mathrm{mm}$ spot size, $0.8 \mathrm{~J} / \mathrm{cm}^{2}$, and a frequency of $10 \mathrm{~Hz}$ in microlens array and a total of approximately 2,000 shots on the pigmented lesion. All laser safety precautions were followed. After treatment, all patients were advised to avoid direct sunlight and use a broad-spectrum sunscreen agent until the end of the study.

\section{Post-laser treatment care}

After laser treatment, each side of the face received different posttreatment methods. After laser treatment, a moisture mask (Kineff Hydracica Calming Mask; Holians, Seoul, Korea) was applied for 30 minutes to moisten the skin. An antioxidant cream containing tocotrienol (Kineff 31 Allday Shield Cream \& 102 Night Renewal Cream; Holians) was applied to the right side of the face. The patients were instructed to apply an antioxidant cream containing tocotrienol to the right side of the face and to apply a topical moisturizer and physical sunscreen to both sides of the face for 4 weeks.

\section{Outcome evaluation}

We evaluated the improvement of skin pigmentation on the face using an automatic skin analysis device (Mark-Vu; PSI Plus Co., Suwon, Korea) with UV light and natural light before and at 4 weeks after treatment. The melasma severity score (MSS) has four grades
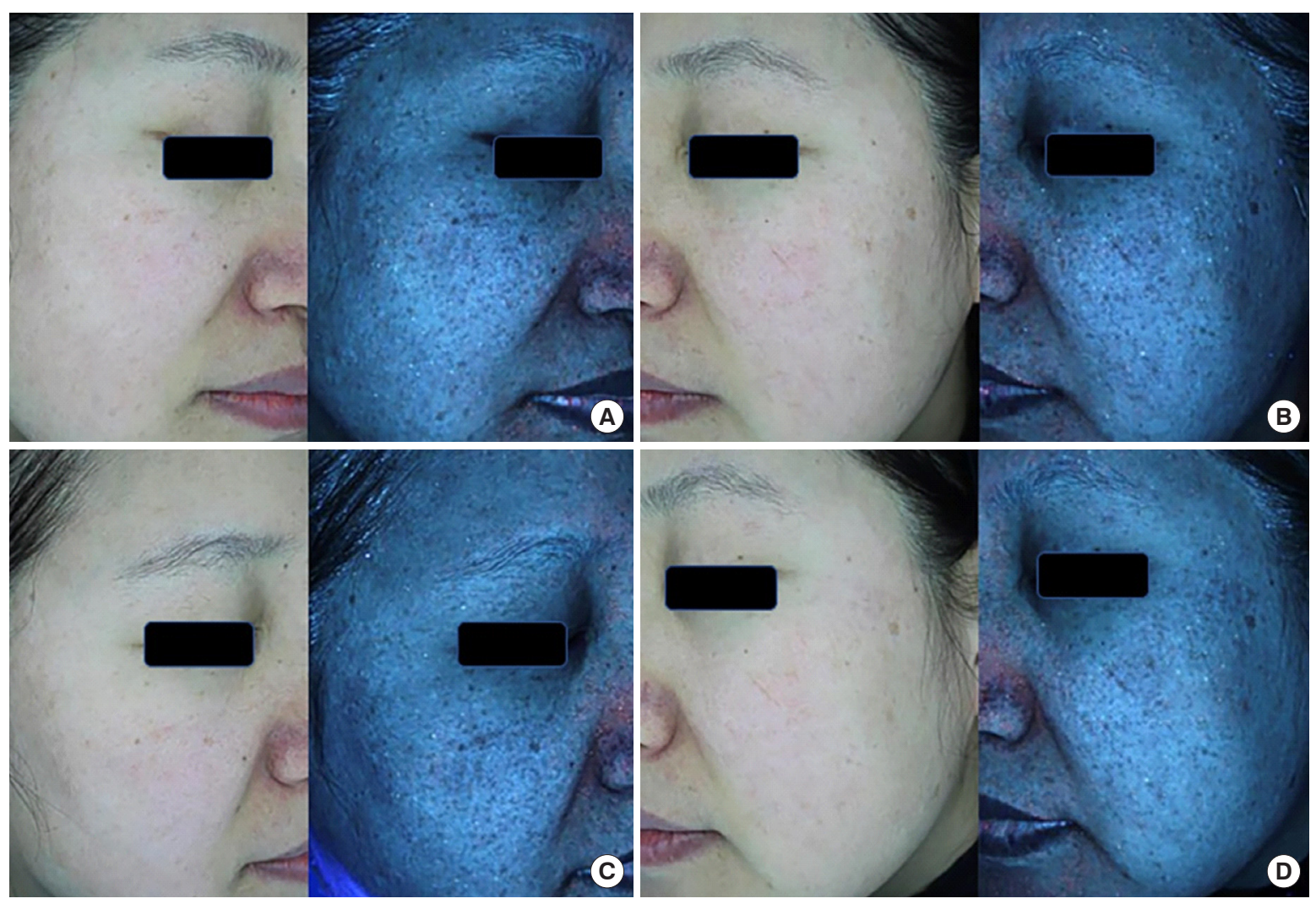

Fig. 2. Clinical and ultraviolet photographs of a 53-year-old female patient with environment-induced skin pigmentation. (A) The right side of the face, to which a combination of picosecond laser and antioxidant cream containing tocotrienol was applied. (B) The left side of the face, to which only picosecond laser treatment was applied. (C) The right side of the face at 4 weeks after laser treatment. (D) The left side of the face at 4 weeks after laser treatment. 
of severity as follows: 0 , equivalent to the surrounding normal skin or with minimal residual pigmentation; 1 , slightly darker than the surrounding normal skin; 2 , moderately darker than the surrounding normal skin; 3 , markedly darker than the surrounding normal skin [18]. The MSS was evaluated by two blinded plastic surgeons (SMN and ESP).

\section{Statistical analysis}

Statistical analyses were performed using SPSS version 20.0 (IBM Corp., Armonk, NY, USA). The Wilcoxon signed-rank test was used to compare the improvement in skin pigmentation and MSS between before laser treatment and 4 weeks after treatment. Statistical significance was set at $\mathrm{P}<0.05$.

\section{RESULTS}

Of the 26 patients with environment-induced skin pigmentation who were treated using a 1064-nm picosecond Nd:YAG laser, 25 were female and one was male. The mean age of the patients was 43.1 years (range, $37-55$ years), and the mean follow-up period was 5 weeks (range, 4-6 weeks) (Table 1). No serious complications, such as infection or skin necrosis, were observed during the followup period.

In this split-face study, we analyzed the efficacy of an antioxidant cream containing tocotrienol after laser treatment (Figs. 1, 2). Skin pigmentation scores (SPSs) were analyzed using an automatic skin analysis device (Mark-Vu). On the right side of the face, treated with antioxidant cream containing tocotrienol after picosecond laser treatment, the SPS was 30 (interquartile range [IQR], 28-31) be-

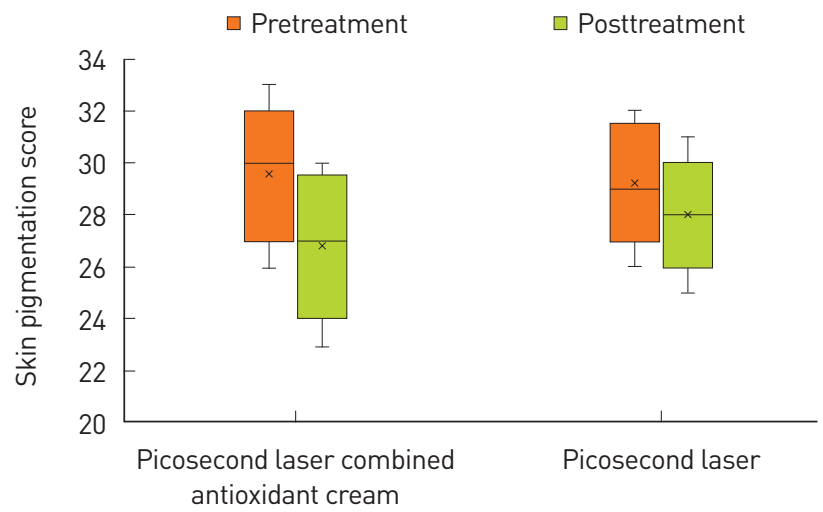

Fig. 3. On the right side of the face ladministered antioxidant cream containing tocotrienol after picosecond laser treatment), the skin pigmentation score (SPS) was 30 (IQR, 28-31) before treatment and 27 (IQR, 25-29) at 4 weeks after treatment. The difference between pretreatment and 4 weeks after laser treatment was statistically significant $(P<0.001)$. On the left side of the face, which only received picosecond laser treatment, the SPS was 29 (IQR, 28-21) before treatment and 28 (IQR, 27-29) at 4 weeks after laser treatment. The difference was statistically significant $(P<0.001)$. IQR, interquartile range. fore treatment and 27 (IQR, 25-29) at 4 weeks after treatment. The difference between pretreatment and 4 weeks after laser treatment was statistically significant $(\mathrm{P}<0.001)$. On the left side of the face, where only picosecond laser treatment was performed, the SPS was 29 (IQR, 28-21) before treatment and 28 (IQR, 27-29) at 4 weeks after laser treatment. The difference was statistically significant $(\mathrm{P}<0.001)$ (Fig. 3). The improvement of the SPS on the right side of the face was 2 (IQR, 2-3), and that on the left side was 1 (IQR, $1-2)$, and the difference in the improvement of SPS between the right and left sides was statistically significant $(\mathrm{P}<0.001)$ (Fig. 4).

In addition, we evaluated the improvement in the MSS between

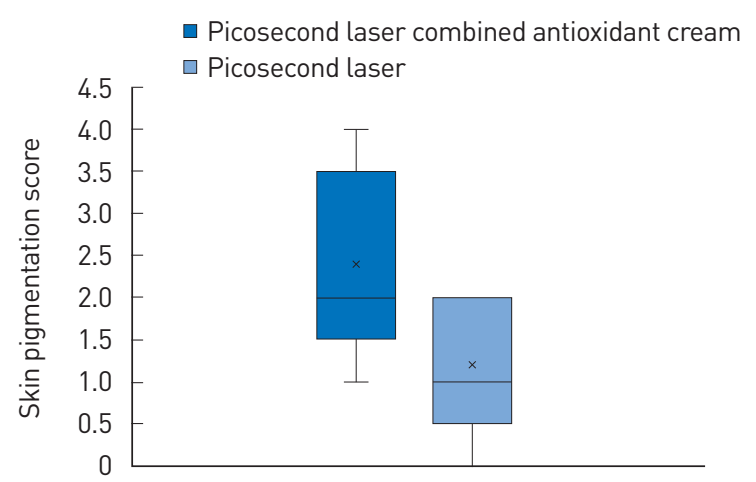

Fig. 4. The improvement in the skin pigmentation score on the right side of the face ladministered antioxidant cream containing tocotrienol after picosecond laser treatment), was 2 (IQR, 2-3) and that on the left side was 1 (IQR, 1-2) at 4 weeks after laser treatment; the difference was statistically significant $(P<0.001)$. IQR, interquartile range.

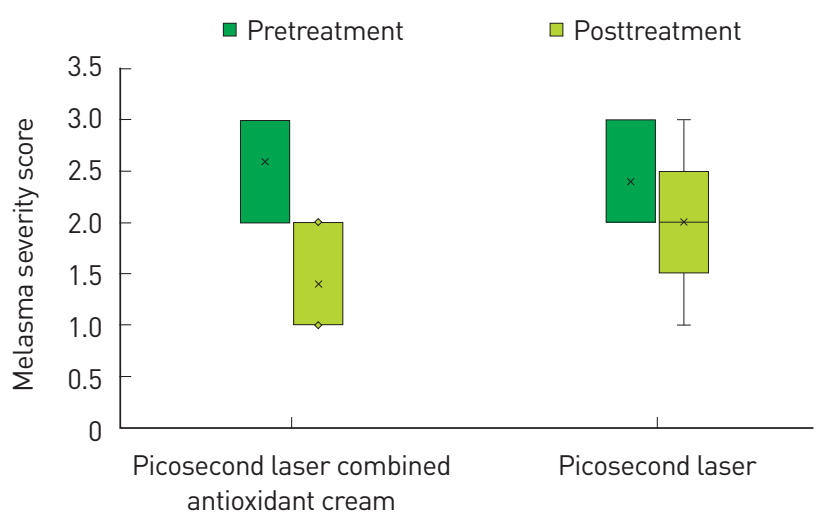

Fig. 5. The melasma severity score (MSS) between the right and left sides of the face. On the right side, the MSS was 3 (IQR, 2-3) before treatment, and $1(I Q R, 1-2)$ at 4 weeks after treatment; the difference was statistically significant $(P<0.001)$. However, on the left side, the MSS was 2 (IQR, 2-3) before treatment and 2 (IQR, 2-2) at 4 weeks after laser treatment; the difference was not significant $(P>0.05)$. IQR, interquartile range. 
the right and left sides of the face. On the right side of the face, the MSS was 3 (IQR, 2-3) before treatment and 1 (IQR, 1-2) at 4 weeks after treatment, and the difference was statistically significant $(\mathrm{P}<$ 0.001). However, on the left side of the face, the MSS was 2 (IQR, 2-3) before treatment and 2 (IQR, 2-2) at 4 weeks after laser treatment; this difference was not significant $(\mathrm{P}>0.05)$ (Fig. 5).

\section{DISCUSSION}

The 1064-nm Q-switched Nd:YAG laser is the most commonly used laser for the treatment of skin pigmentation lesions in Asian populations, and its outcomes have been verified to be satisfactory $[19,20]$. However, picosecond lasers have recently been developed. These have a very short pulse duration with the same energy, thus reducing the photothermal effect, while increasing the photomechanical effect. They can produce significantly smaller pigment particles and can easily phagocytose macrophages [21].

Environment-induced skin pigmentation, such as melasma and solar lentigines, are common hyperpigmentation disorders in the Asian population [11]. These conditions most commonly occur in middle-aged women and usually present as symmetrical, irregular hypermelanosis on the bilateral central cheeks [11]. The pathogenesis of skin pigmentation is unclear, although it may be mainly related to UV radiation. Skin pigmentation has a complex pathway that primarily occurs in melanocytes, which are dendritic cells that synthesize melanin in melanosomes. Melanosomes produce supranuclear caps that protect epidermal cells from damage by limiting the penetration of UV radiation and scavenging reactive oxygen species [17]. Melanogenesis is regulated by tyrosinase, tyrosinaserelated protein-1, and tyrosine-related protein-2 (TYR, TYRP1, and TYRP2). The control and regulation of these enzymes are important processes for decreasing skin pigmentation [22]. Makpol et al. [17] reported that tocotrienol and tocopherol treatment reduced the expression of TYR, TYRP1, and TYRP2, leading to a significant decrease in melanin content, which is determined by the amount of melanin synthesis in melanocytes. In particular, the anti-melanogenic properties of tocotrienol may play an important role in inhibiting tyrosinase activity [17].

Hydroquinone and retinoids have been suggested to improve skin pigmentation. However, the most common complications of hydroquinone and retinoids are irritation, erythema, and desquamation [18]. It was reported that in rare cases, application of hydroquinone led to further hyperpigmentation in patients with darker skin [23]. Vitamin C derivatives are usually used to treat skin pigmentation and post-inflammatory hyperpigmentation. Several studies reported that ascorbic acid reduced dopaquinone, which inhibits tyrosine-dependent melanogenesis [24,25].

In our split-face study, we evaluated the efficacy of an antioxidant cream containing tocotrienol after picosecond laser treatment. The SPS of both sides of the face significantly improved at 4 weeks after laser treatment compared to the pretreatment values. The right side of the face, to which antioxidant cream containing tocotrienol was applied after picosecond laser treatment exhibited a greater improvement in SPS than the left side, to which only picosecond laser treatment was administered. The differences in skin pigmentation improvement were statistically significant. We also compared the MSS score on both sides of the face before laser treatment and 4 weeks after picosecond laser treatment. On the right side of the face, the change in the MSS score between pretreatment and 4 weeks after laser treatment was statistically significant. However, on the left side of the face, the change in the MSS score was not significant. Based on our study, we conclude that antioxidant cream containing tocotrienol increases the therapeutic effect of environment-induced skin pigmentation, although the 1064-nm picosecond laser plays an important role in the treatment of skin pigmentation. However, our study has some limitations, such as a small number of patients, a relatively short-term follow-up period, and the inclusion of only Asian individuals with Fitzpatrick skin types III-IV. Therefore, we plan to conduct further studies with a larger sample size, a longer-term follow-up period, and the inclusion of Caucasian individuals.

Despite these limitations, the application of an antioxidant cream containing tocotrienol enhanced the therapeutic effects of a 1064$\mathrm{nm}$ picosecond laser for the treatment of environment-induced skin pigmentation.

\section{NOTES}

\section{Conflict of interest}

Eun Soo Park is an editorial board member of the journal but was not involved in the peer reviewer selection, evaluation, or decision process of this article. No other potential conflicts of interest relevant to this article were reported.

\section{Ethical approval}

The study was approved by the Institutional Review Board of Soonchunhyang University Bucheon Hospital (IRB No. 2021-06-015) and performed in accordance with the principles of the Declaration of Helsinki.

\section{Patient consent}

The patients provided written informed consent for the publication and the use of their images.

\section{ORCID}

Moon Seok Kang

Jun Hyun Kim

Seung Min Nam

Eun Soo Park https://orcid.org/0000-0001-7072-6091

https://orcid.org/0000-0003-0410-2680

https://orcid.org/0000-0001-7865-5013

https://orcid.org/0000-0003-2966-9122 


\section{REFERENCES}

1. Munavalli GS, Weiss RA, Halder RM. Photoaging and nonablative photorejuvenation in ethnic skin. Dermatol Surg 2005;31(9 Pt 2):125060 .

2. Cecchi L, D’Amato G, Annesi-Maesano I. External exposome and allergic respiratory and skin diseases. J Allergy Clin Immunol 2018;141: 846-57.

3. Dreno B, Tan J, Kang S, et al. How people with facial acne scars are perceived in society: an online survey. Dermatol Ther (Heidelb) 2016;6: 207-18.

4. Krutmann J, Bouloc A, Sore G, et al. The skin aging exposome. J Dermatol Sci 2017;85:152-61.

5. Vierkotter A, Schikowski T, Ranft U, et al. Airborne particle exposure and extrinsic skin aging. J Invest Dermatol 2010;130:2719-26.

6. Bansal C, Naik H, Kar HK, et al. A comparison of low-fluence 1064nm Q-switched Nd: YAG laser with topical 20\% azelaic acid cream and their combination in melasma in Indian patients. J Cutan Aesthet Surg 2012;5:266-72.

7. Lee CN, Kim YJ, Lee HS, et al. Effects of Q-switched and long-pulsed $1064 \mathrm{~nm}$ Nd:YAG laser on enlarged facial pores. Photodermatol Photoimmunol Photomed 2009;25:328-30.

8. Lee MC, Chang CS, Huang YL, et al. Treatment of melasma with mixed parameters of 1,064-nm Q-switched Nd:YAG laser toning and an enhanced effect of ultrasonic application of vitamin C: a split-face study. Lasers Med Sci 2015;30:159-63.

9. Park KY, Kim DH, Kim HK, et al. A randomized, observer-blinded, comparison of combined 1064-nm Q-switched neodymium-doped yttrium-aluminium-garnet laser plus 30\% glycolic acid peel vs. laser monotherapy to treat melasma. Clin Exp Dermatol 2011;36:864-70.

10. Jones CE, Nouri K. Laser treatment for pigmented lesions: a review. J Cosmet Dermatol 2006;5:9-13.

11. Lee MC, Lin YF, Hu S, et al. A split-face study: comparison of picosecond alexandrite laser and Q-switched Nd:YAG laser in the treatment of melasma in Asians. Lasers Med Sci 2018;33:1733-8.

12. Alabdulrazzaq $\mathrm{H}$, Brauer JA, Bae YS, et al. Clearance of yellow tattoo ink with a novel 532-nm picosecond laser. Lasers Surg Med 2015;47: 285-8.

13. Brauer JA, Kazlouskaya V, Alabdulrazzaq H, et al. Use of a picosecond pulse duration laser with specialized optic for treatment of facial acne scarring. JAMA Dermatol 2015;151:278-84.
14. Khetarpal S, Desai S, Kruter L, et al. Picosecond laser with specialized optic for facial rejuvenation using a compressed treatment interval. Lasers Surg Med 2016;48:723-6.

15. Funasaka Y, Chakraborty AK, Komoto M, et al. The depigmenting effect of alpha-tocopheryl ferulate on human melanoma cells. Br J Dermatol 1999;141:20-9.

16. Kamei Y, Otsuka Y, Abe K. Comparison of the inhibitory effects of vitamin $\mathrm{E}$ analogues on melanogenesis in mouse B16 melanoma cells. Cytotechnology 2009;59:183-90.

17. Makpol S, Jam FA, Rahim NA, et al. Comparable down-regulation of TYR, TYRP1 and TYRP2 genes and inhibition of melanogenesis by tyrostat, tocotrienol-rich fraction and tocopherol in human skin melanocytes improves skin pigmentation. Clin Ter 2014;165:e39-45.

18. Kim J, Kim J, Lee YI, et al. Effect of a topical antioxidant serum containing vitamin C, vitamin E, and ferulic acid after Q-switched 1064$\mathrm{nm}$ Nd:YAG laser for treatment of environment-induced skin pigmentation. J Cosmet Dermatol 2020;19:2576-82.

19. Kim JE, Chang SE, Yeo UC, et al. Histopathological study of the treatment of melasma lesions using a low-fluence Q-switched 1064-nm neodymium:yttrium-aluminium-garnet laser. Clin Exp Dermatol 2013; 38:167-71.

20. Kim JH, Kim H, Park HC, et al. Subcellular selective photothermolysis of melanosomes in adult zebrafish skin following 1064-nm Q-switched Nd:YAG laser irradiation. J Invest Dermatol 2010;130:2333-5.

21. Choi YJ, Nam JH, Kim JY, et al. Efficacy and safety of a novel picosecond laser using combination of 1064 and $595 \mathrm{~nm}$ on patients with melasma: a prospective, randomized, multicenter, split-face, $2 \%$ hydroquinone cream-controlled clinical trial. Lasers Surg Med 2017;49:899907.

22. Gaggioli C, Busca R, Abbe P, et al. Microphthalmia-associated transcription factor (MITF) is required but is not sufficient to induce the expression of melanogenic genes. Pigment Cell Res 2003;16:374-82.

23. Torok HM, Jones T, Rich P, et al. Hydroquinone $4 \%$, tretinoin $0.05 \%$, fluocinolone acetonide $0.01 \%$ : a safe and efficacious 12 -month treatment for melasma. Cutis 2005;75:57-62.

24. Ando H, Kondoh H, Ichihashi M, et al. Approaches to identify inhibitors of melanin biosynthesis via the qualit,hjhgy control of tyrosinase. J Invest Dermatol 2007;127:751-61.

25. Stamford NP. Stability, transdermal penetration, and cutaneous effects of ascorbic acid and its derivatives. J Cosmet Dermatol 2012;11:310-7. 